\title{
Estimating the Force of Infection for Dengue Virus Using Repeated Serosurveys, Ouagadougou, Burkina Faso
}

Jacqueline K. Lim, Mabel Carabali, Tansy Edwards, Ahmed Barro, Jung-Seok Lee, Desire Dahourou, Kang Sung Lee, Teguewende Nikiema, Mee Young Shin, Emmanuel Bonnet, Therese Kagone, Losseni Kaba, Suk Namkung, Paul-André Somé, Jae Seung Yang, Valéry Ridde, In-Kyu Yoon, Neal Alexander, Yaro Seydou

\begin{abstract}
Because of limited data on dengue virus in Burkina Faso, we conducted 4 consecutive age-stratified longitudinal serologic surveys, $\approx 6$ months apart, among persons 1-55 years of age, during June 2015-March 2017, which included a 2016 outbreak. The seroconversion rate before the serosurvey enrollment was estimated by binomial regression, taking age as the duration of exposure, and assuming constant force of infection (FOI) over age and calendar time. We calculated FOI between consecutive surveys and rate ratios for potentially associated characteristics based on seroconversion using the duration of intervals. Among 2,897 persons at enrollment, $66.3 \%$ were IgG-positive, and estimated annual FOI was $5.95 \%$. Of 1,269 enrollees participating in all 4 serosurveys, 438 were IgGnegative at enrollment. The annualized FOI ranged from $10 \%$ to $20 \%$ (during the 2016 outbreak). Overall, we observed high FOI for dengue. These results could support decision-making about control and preventive measures for dengue.
\end{abstract}

Author affiliations: International Vaccine Institute, Seoul, South Korea (J.K. Lim, M. Carabali, J.-S. Lee, K.S. Lee, M.Y. Shin,

S. Namkung, J.S. Yang); London School of Hygiene and Tropical Medicine, London, UK (J.K. Lim, T. Edwards,

N. Alexander); Centre MURAZ, Bobo-Dioulasso, Burkina Faso

(D. Dahourou, T. Nikiema, T. Kagone, Y. Seydou); McGill

University, Montreal, Quebec, Canada (M. Carabali); Action,

Gouvernance, Intégration, Renforcement Program Equité,

Ouagadougou, Burkina Faso (A. Barro, P.-A. Somé); Institut de

Recherché en Sciences de la Santé, Ouagadougou

(D. Dahourou); Institute for Research on Sustainable

Development, Université de Paris, Paris, France (E. Bonnet,

V. Ridde); Centre National de Transfusion Sanguine,

Ouagadougou (L. Kaba); Coalition for Epidemic Preparedness

Innovations, Washington, DC, USA (I.-K. Yoon)

DOI: https://doi.org/10.3201/eid2701.191650
Dengue fever is a mosquitoborne disease caused by 4 related but antigenically distinct dengue viruses (DENVs), serotypes $1-4$. Annually, $\approx 50-100$ million cases of dengue are reported worldwide, with 20,000 deaths (1). Aedes mosquitoes and dengue cases were documented in Africa as early as 1823, and cases have since been reported in 34 countries in Africa (2). In Burkina Faso, since the first outbreak in 1925, there have been multiple others (2), including in 2013, 2016, and 2017 (3-5). The 2016 outbreak included 1,061 dengue rapid-diagnostic test (RDT) positive cases and 15 deaths in the capital, Ouagadougou, with a reported case-fatality rate (CFR) of $1.2 \%(4,6)$. The 2017 outbreak included 5,773 RDT-positive cases and 18 deaths throughout the country, for a CFR of $0.2 \%$ (5). These repeated outbreaks suggest a considerable dengue burden in the country.

Despite this burden, data on dengue seroprevalence and force of infection (FOI), the rate at which initial or heterotypic infections are acquired, are scarce in Burkina Faso and Africa (7). In terms of seroprevalence in Burkina Faso, 1 study found the dengue IgG seroprevalence among 683 pregnant women and blood donors to be $26.3 \%$ in rural and $36.5 \%$ in urban settings in 2003-2004 (8). To define DENV transmission in Burkina Faso, we conducted 4 serologic surveys in the same study participants in Ouagadougou during 2015-2017. The study targeted 3 objectives. First, we measured seroprevalence of DENV by IgG positivity at enrollment, serosurvey 1 (S1). Second, we estimated age-specific annual FOI, measured by seroconversion in the repeated follow-up surveys (S2-S4). Last, because a dengue outbreak occurred in 2016, between the third and fourth serosurveys, we identified and compared demographic and clinical characteristics associated with DENV seroconversion in the outbreak and nonoutbreak periods. 


\section{Methods}

\section{Study Area and Population}

We selected the study area based on data, including seroprevalence and modeling results, available in the literature and existing research infrastructure (9-11). Ouagadougou is the capital of Burkina Faso in West Africa; most of the population resides in urban settings $(12,13)$. The rainy season is May-October. The serosurveys were conducted in a defined catchment population of 100,000 residents. The resident population in Ouagadougou is stable, with an annual rate of migration of only $4.1 \%$ and $>80 \%$ of residents owning their homes (14).

\section{Study Design}

We conducted 4 serosurveys, each $\approx 6$ months apart. The age-stratified sample of $\approx 3,000$ residents $1-55$ years of age, $80 \%<35$ years of age, reflected the age distribution of the general population of Ouagadougou (9). In 6 preselected sectors, we randomly selected households on the basis of existing census data; all eligible household members were offered enrollment. To reach the needed sample size, if members of the initially invited household declined, we invited a neighboring household to enroll. We conducted a short interview and collected blood samples (9). Test results were shared with the participants and we followed the same procedures with the same participants for the 3 subsequent serosurveys.

\section{Laboratory Testing Algorithm}

We tested samples using a Panbio Dengue IgG Indirect ELISA test (Abbott Diagnostics, https://www. abbott.com), as described elsewhere (9). Following the manufacturer's guidelines, we set the IgG threshold for positivity at an index value of 1.1, to detect levels resulting from past or recent infections of any serotype. An index value of 0.9-1.1 was classified equivocal (requiring repeated testing), and $<0.9$ was considered negative. We considered seroconversion of dengue IgG between the pretransmission and posttransmission surveys to result from dengue infection.

\section{Statistical Analysis}

\section{Characteristics by Dengue IgG Status at Enrollment}

We present a descriptive summary by dengue IgG status at enrollment (seropositive vs. seronegative). We used $\chi^{2}$ or Fisher exact tests to make categorical pair-wise comparisons across dengue status. Continuous variables were compared using Student $t$-test or analysis of variance.

\section{FOI Calculations}

DENV infection can occur with any of the 4 serotypes and, assuming lifelong acquired homotypic immunity, we estimated the FOI based on IgG seropositivity status $(15,16)$. However, because IgG ELISA tests cannot distinguish among the 4 serotypes, infection in this analysis refers to seroconversion to any DENV serotype (15). We used binomial regression with a complementary log-log link function (17-20). In part A of the FOI analysis, in which data from the enrollment serosurvey were analyzed, we estimated the average FOI over each participant's lifetime using age as the time at risk. In part B of the FOI analysis, using data from the subset of participants who contributed to all 4 serosurveys, we estimated the FOI between consecutive surveys. We considered participants who were initially seronegative to be at risk for seroconversion and used the interval between consecutive surveys as the time at risk. We provide details of FOI calculations in the Appendix (https://wwwnc.cdc. gov/EID/article/27/1/19-1650-App1.pdf).

\section{Seroconversion Rate Ratios}

For the between-survey analyses, we estimated seroconversion rate ratios (RRs) using binomial regression models with the log time of the actual duration of each participant's interval (i.e., time between consecutive surveys for that person) for potential risk factors, including age, sex, neighborhood, level of education, occupation, any known previous dengue infection, yellow fever (YF) vaccination history, and any self-reported signs and symptoms during the particular interval. As a sensitivity analysis, seroconversion RRs were estimated for consecutive paired results, irrespective of results from other surveys. For example, if a participant was IgG-negative at S2, then IgG-positive at S3, we considered this seroconversion between S2 and S3, even if the person had been IgGpositive at $\mathrm{S} 1$.

S1-S2 covered the nonoutbreak rainy season in 2015 , S2-S3 covered the nonoutbreak nonrainy season in 2016, and S3-S4 covered the 2016 outbreak. To assess how demographic and clinical characteristics are associated with DENV seroconversion and the difference in patterns in the outbreak (S3-S4) compared with those in nonoutbreak periods (S1S2 and S2-S3), we compiled a descriptive summary of demographic and clinical characteristics for participants at risk (IgG-negative) at each serosurvey, broken down between participants who had or had not seroconverted by the subsequent serosurvey. All analyses were performed using SAS version 9.4 (https://www.sas.com). 


\section{Ethics Considerations}

The study protocol received ethics approvals from the institutional review boards of the International Vaccine Institute, the London School of Hygiene and Tropical Medicine, the National Ethical Committee for Health Research of Burkina Faso, and the Ethics Committee of the Centre Hospitalier de l'Université de Montréal at University of Montreal. We obtained written consent forms from each participant $\geq 18$ years old. For participants 8-17 years old, we obtained an assent form from the participant and an informed consent from $\geq 1$ parent or legal guardian. For participants $\leq 7$ years old, we obtained an informed consent from $\geq 1$ parent or legal guardian.

\section{Results}

We obtained complete demographic (age and neighborhood) and laboratory data for 2,897 of 3,026 participants (Figure 1). At enrollment (S1), in June 2015, $1,920(66.3 \%)$ of 2,897 participants were IgG positive. At S2, in December 2015, 1,417 (67.2\%) of 2,109 participants were IgG positive. At S3, in May 2016, 1,400 $(66.5 \%)$ of 2,106 participants were IgG positive. At S4, in March 2017, 1,121 (67.9\%) of 1,651 participants were IgG positive.

\section{Characteristics by Dengue IgG Status at Enrollment}

Based on data from the 2,897 participants with complete information, $82 \%$ of 1 -4-year-olds and $65 \%$ of 5-9-year-olds were IgG negative and therefore at risk of infection at the start of the study (Table 1).
IgG positivity increased with age, so that by age 26 , IgG positivity reached $80 \%$ (Figure 2 ). The binomial regression based on IgG positivity by age at enrollment, assuming the FOI was constant over ages and calendar time (part A), resulted in an annual FOI of 5.95\% (95\% CI 5.66-6.24) (Figure 2).

\section{Annual FOI in IgG-Negative Participants Who Contributed to All 4 Serosurveys}

In part B, we calculated annualized FOI among participants who had been IgG negative at the preceding serosurvey using binomial regression with the intersurvey interval, calculated in years, as the time at risk. For the interval S1-S2, FOI per year was $14.0 \%$ (95\% CI $9.5 \%-18.4 \%)$; for the interval S2-S3, 9.6\% (95\% CI $5.4 \%-13.8 \%)$; and for the interval S3-S4, 20.3\% (95\% CI 16.1\%-24.5\%) (Figure 1). The mean duration was 0.53 years ( $\approx 6$ months) for interval S1-S2; 0.44 years for S2-S3; and 0.84 years for S3-S4. Age-specific annual FOIs were calculated and older age groups with $<5$ seronegative participants were merged. For intervals S1-S2 and S2-S3, FOI was higher for older participants (Figure 1). For interval S3-S4, high FOIs of $30 \%$ per year were found in participants 15-19 and 25-29 years of age.

\section{Seroconversion RRs from Outbreak Versus Nonoutbreak Intervals}

We analyzed data from each pair of surveys, not restricted to data from participants in all 4 surveys, to examine differences in dengue seroconversion

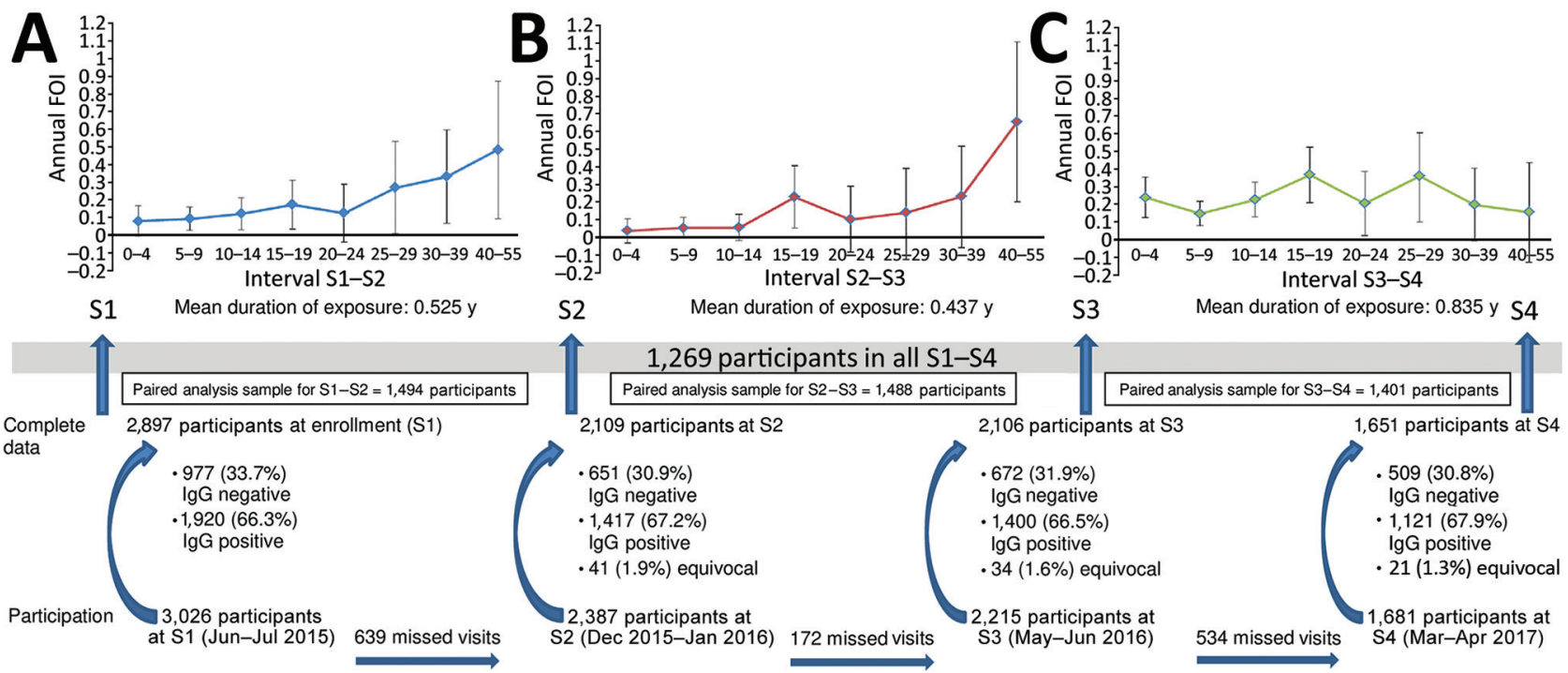

Figure 1. Flowchart of participant enrollment and graphs of annual force of infection rates during a rainy season (A), a nonrainy season (B), and the 2016 dengue outbreak (C) in study of force of infection for dengue virus, Ouagadougou, Burkina Faso, June-July 2015. Labels along x-axes (e.g., 0-4) indicate age ranges in years. Error bars indicate $95 \% \mathrm{Cls}$. To be considered complete, records had to contain lab results and basic demographic information. Seroconversion rates in the paired analyses were based solely on results from one survey to the next (e.g., S2-S3). Dengue serostatus in previous or subsequent surveys was not considered. S, serosurvey. 
during outbreak versus nonoutbreak intervals. During nonoutbreak interval S1-S2 (analysis sample $=1,494$ ), $33(7.3 \%)$ of 455 participants at risk (i.e., IgG negative at
S1), showed seroconversion. During nonoutbreak interval S2-S3 (analysis sample = 1,488), 23 (5.2\%) of 443 participants at risk (i.e., IgG negative at S2), showed

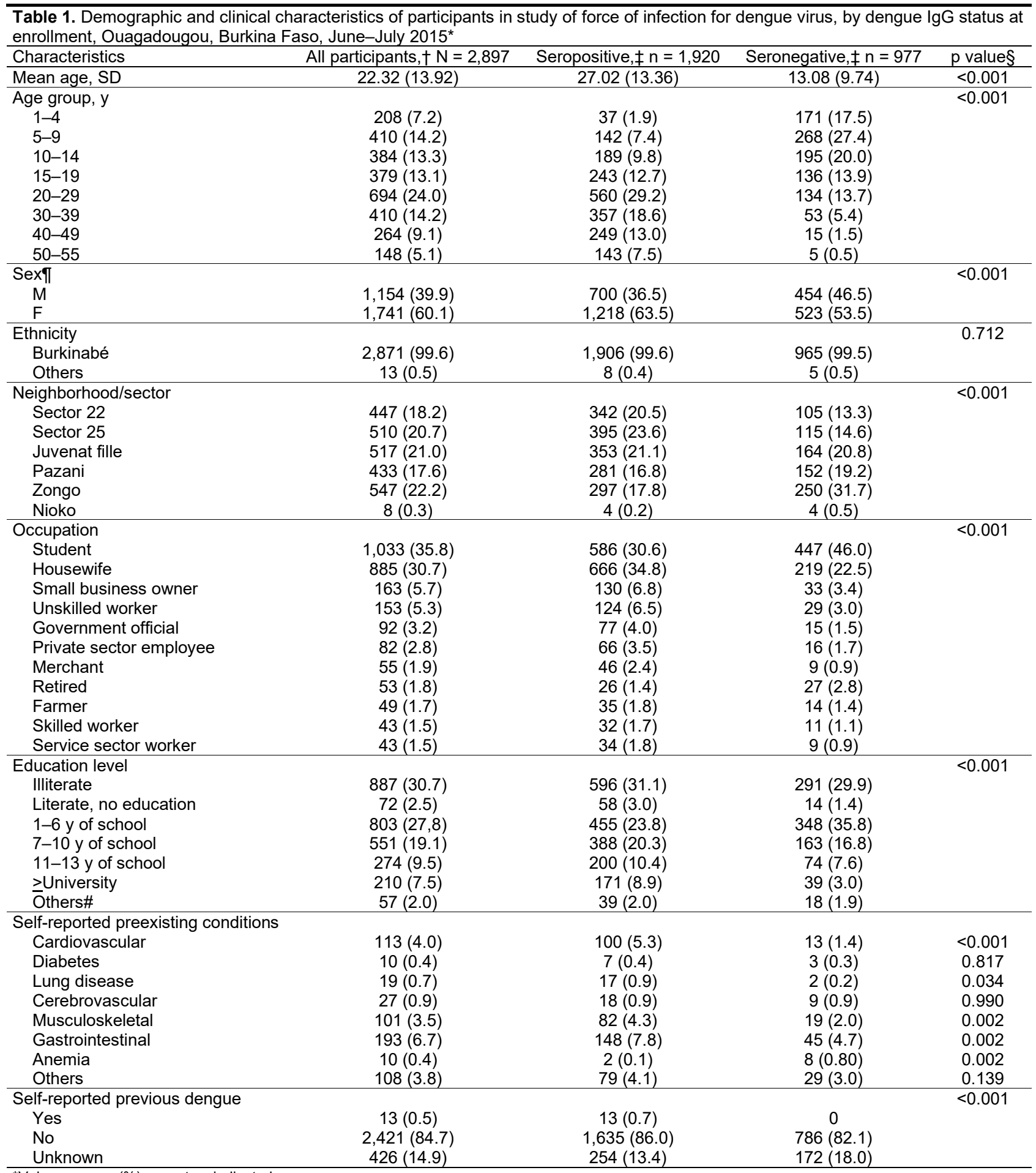

*Values are no. (\%) except as indicated.

†Total participants enrolled at serosurvey 1.

†Results of IgG indirect ELISA among participants at serosurvey 1.

$\S p$ values based on $X^{2}$ test.

\2 individuals with missing information on sex.

\#Religious and other informal education. 


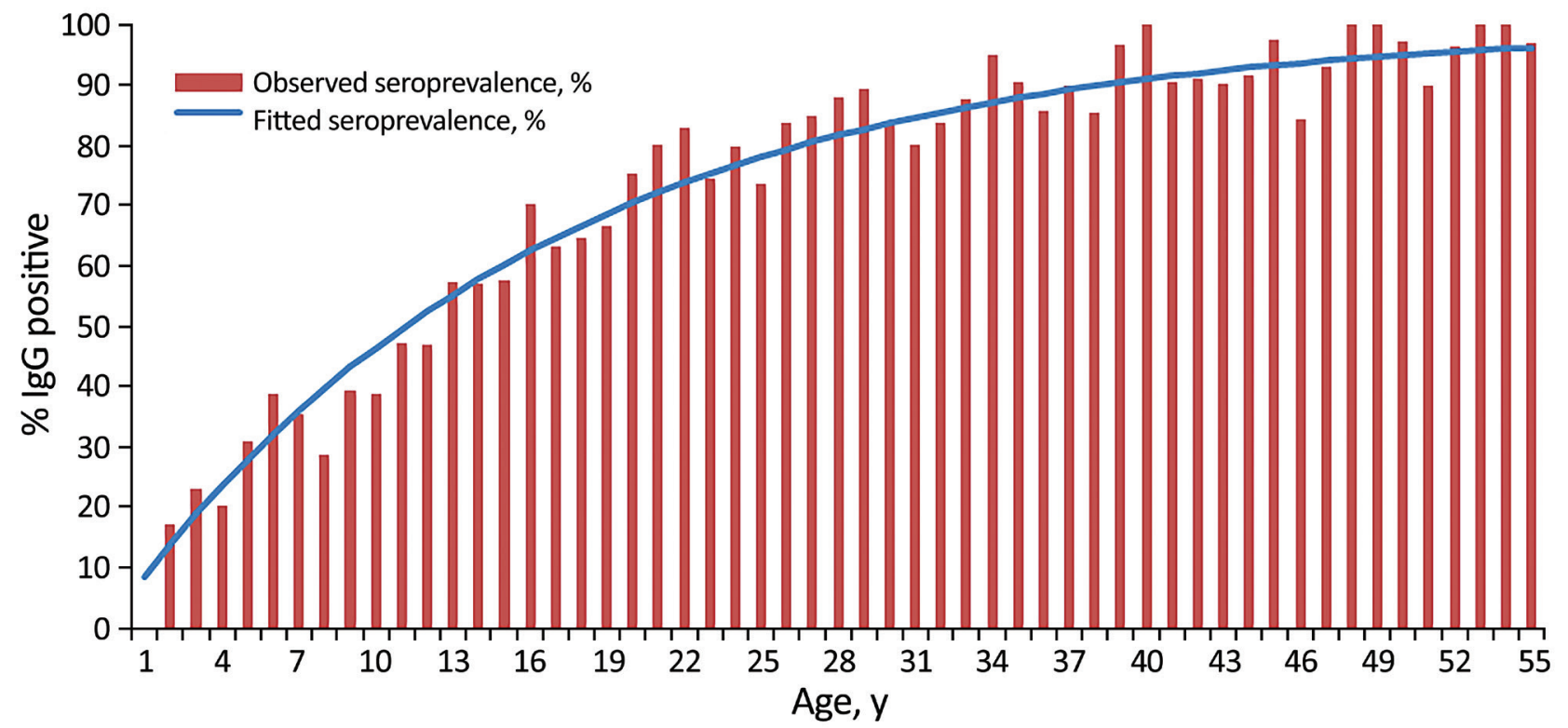

Figure 2. Seroprevalence, measured by IgG ELISA, of dengue IgG by age at enrollment and fitted prevalence using the FOI per year in study of force of infection for dengue virus, Ouagadougou, Burkina Faso, June-July 2015. Graph shows observed seroprevalence at enrollment among all 2,897 participants and fitted seroprevalence using FOI. In the FOI analysis part A, the FOI per year was 0.0595 $(95 \% \mathrm{Cl} 0.0566-0.0624)$, estimated by binomial regression, with the assumption of constant risk across ages and calendar time prior to the enrollment serosurvey, and a complementary log-log link, with log(midpoint of age) as an offset. The intercept is interpreted as the logarithm of the FOI. FOI, force of infection.

seroconversion. During outbreak interval S3-S4 (analysis sample $=1,401) 78(17.1 \%)$ of 455 participants at risk (i.e., IgG negative at S3), showed seroconversion. We compiled demographic and clinical characteristics of participants with IgG seroconversion compared with participants who remained IgG negative during each interval (Table 2, https:/ / wwwnc.cdc.gov/EID/ article/27/1/19-1650-T2.htm).

To assess how these variables might be associated with changes in rates of seroconversion, we estimated RRs of seroconversion during the intervals. We found that older age was positively associated with an increased rate of seroconversion. Compared with those 1-4 years of age, participants $25-55$ years of age had higher seroconversion over S1-S2 [RR 4.1 (95\% CI 1.4-15.0)]; both 15-24-year-old participants [RR 4.6 (95\% CI 1.4-17.4)] and 25-55-year-old participants [RR 9.1 (95\% CI 12.9-34.2)] had higher seroconversion over S2-S3 (Table 3).

\section{Discussion}

Our study provides data on population-based seroprevalence and rates of DENV seroconversion that may help with assessing the largely undocumented burden of dengue fever in Africa. The dengue burden in Africa has been conjectured to be similar to that in the Americas but has been largely unrecognized and masked by other illnesses with similar symptoms $(21,22)$. In particular, the major strength of this study was that we longitudinally followed the same participants using 4 repeated serosurveys to measure the FOI of dengue in Burkina Faso.

Although IgG ELISA test results might be influenced by cross-reactivity with different flaviviruses, our estimate of seroprevalence was comparable to prevalence estimates from other studies, all tested using IgG ELISA: $61 \%$ of participants 1-65 years of age in Colombia (25); $83.1 \%$ in participants $15-19$ years of age in Tahiti (26); 74\% in participants in a low socioeconomic area in Recife, Brazil (27); and 68.7\% in participants in Salvador, Brazil (28). The overall proportion of IgG-positive participants remained similar across the surveys, although it was highest at S4 $(68 \%)$. The small increase in seropositivity despite an outbreak between S3 and S4 may have resulted from different participants being lost to follow-up at each consecutive survey. Such losses were substantial during the S1-S2 and S3-S4 intervals. The mean age of participants lost during S1-S2 was 20.7 years (95\% CI 19.3-22.0) and was 23.7 (95\% CI: 22.5-24.9) during S3S4. The mean age of the participants was 22.2 years (95\% CI 21.6-22.9) during S1-S2 and 21.6 (95\% CI 21.0-22.2) during S3-S4. For S1-S2, the mean age was not significantly different between nonparticipants 
and remaining participants, but lost participants were significantly older for S3-S4; participants lost at follow-up might have tended to be IgG positive, plausibly resulting in underestimating IgG positivity at the follow-up survey. While decreasing representativeness is a limitation, we based FOI calculations on seroconversion meaning they likely would not have been substantially affected. We found apparent seroreversion from seropositive to seronegative in $<3 \%$ of the paired samples: 29 during S1-S2, 14 during S2-S3; and 38 during S3-S4. However, we could not distinguish whether such cases were due to test errors or waning immunity.

Data from the baseline serosurvey in this study (part A) resulted in an annual FOI of 6.0\%, although this assumes that it was constant over age and calendar time. This finding is comparable with estimates from other regions, such as Sri Lanka at $14 \%$ per year (15); Colombia at 8.7\% (22); and a low socioeconomic area in Recife, Brazil, at 5.3\% (25). Our model seems to capture the increase in baseline seroprevalence with age (Figure 1). However, any given age profile in a cross-sectional study can result from different combinations of incidence varying over age or over calendar time (27). Therefore, the estimated FOI from the baseline survey is subject to more limitations than FOIs estimated from the paired surveys. Nonetheless, our data support that dengue may be a bigger public health problem in Africa than is currently recognized.

From the repeat surveys (part B) for which we knew the exact duration of the intervals, we could measure the FOI more directly without assuming a constant value over age, providing a more accurate estimate of the magnitude of transmission. These estimates were higher than the $6 \%$ annual FOI based on the enrollment survey. Among 1,269 participants with IgG results from all 4 serosurveys, annual FOIs were $14 \%$ for S1-S2, $10 \%$ for S2-S3, and 20\% for S3-S4. Testing of paired samples showed that 7.3\% (33/455) seroconverted during S1-S2, 5.2\% (23/443) during S2-S3, and $17.1 \%(78 / 455)$ during S3-S4. As we expected, the annualized estimates of rates were $\approx 2$ times the simple proportions for the first 2 intervals. For the third interval (S3-S4), the annualized FOI was $\approx 1.5$ times (12/8 months) the proportion seroconverting, because the conversion between proportions and rates is less linear for higher values.

The paired survey analyses did not require an assumption of constant FOI before the study and showed that FOI varied markedly across time within the study. In particular, a high annualized FOI (20\%) was observed between S3-S4, coinciding with a major outbreak. However, even during nonoutbreak intervals, we found considerable dengue transmission; annualized FOIs ranged from 10\% (in a nonrainy

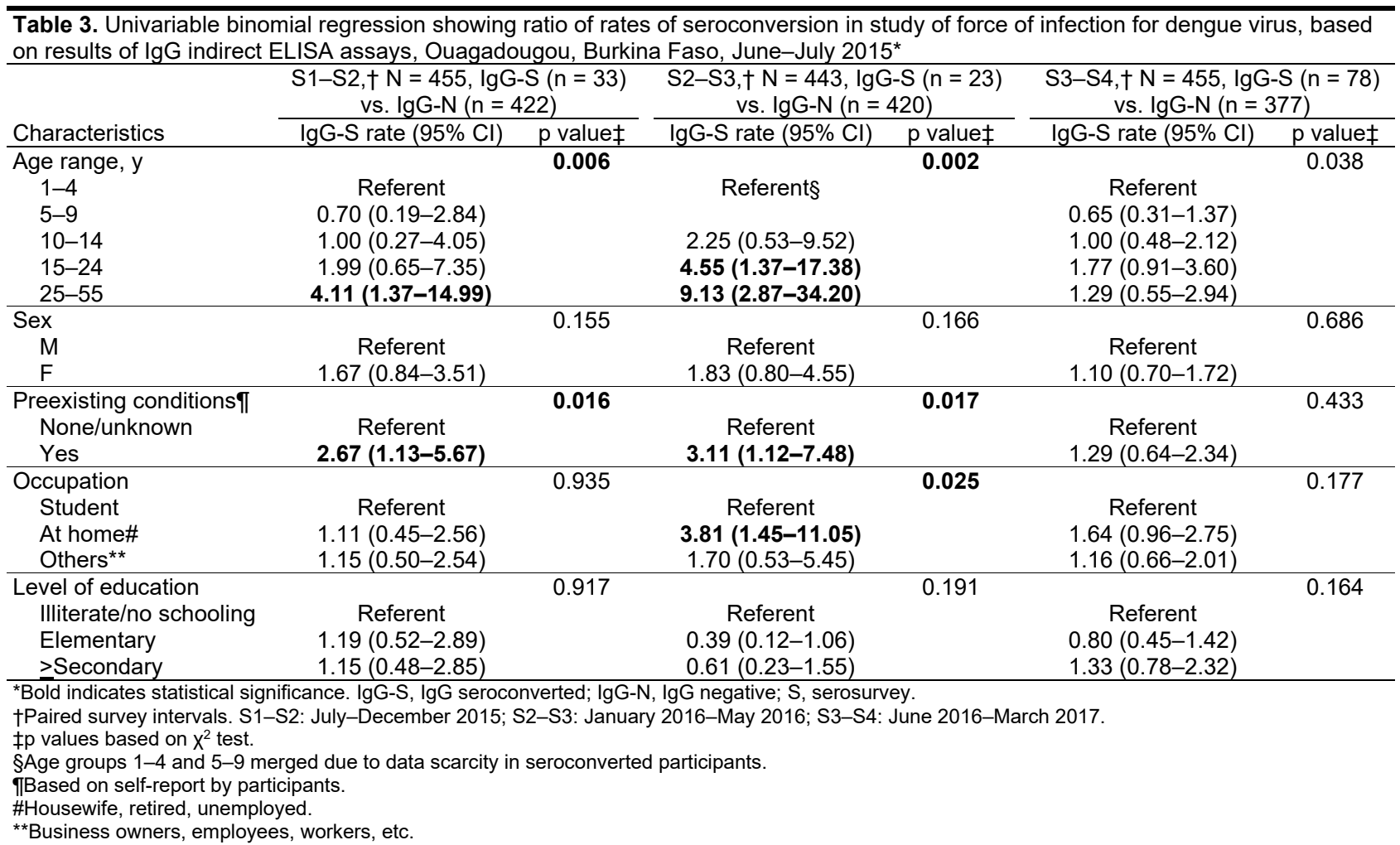


season, such as $\mathrm{S} 2-\mathrm{S} 3$ ) to $14 \%$ (in a rainy season, S1-S2) (28). Little comparable information from the region is available, but these rates are comparable to the $8.7 \%$ per year reported from Colombia (23).

These annual rates of infection, measured by seroconversion, may include both initial and subsequent heterotypic infection, because these are indistinguishable by IgG ELISA. However, in our analysis, the influence of past infection is minimal, because we focused on participants with IgG-negative status at enrollment for calculations of FOIs between surveys. This study was unable to distinguish participants with single or multiple infections during the study period. Furthermore, among those with multiple infections, cyclical dominance among DENV serotypes is observed in regions with better-documented dengue endemicity $(29,30)$. However, cocirculation of multiple DENV serotypes in Burkina Faso has yet to be fully demonstrated. Thus, in our study, we calculated an overall FOI, for the totality of serotypes $(20,31)$. Still, we recognize the need for more in-depth analyses using neutralization assays to understand serotype-specific transmission patterns in Africa.

In part B of the study, without having to assume that risk is constant over age, we found that FOI was higher in older than in younger participants, although these findings are based on small numbers, given that most people had already seroconverted at younger ages. FOI increased with age in the intervals of the nonoutbreak times (S1-S2 and S2-S3), which has also been found in Colombia (23). Our data are from IgG seroconversion rather than clinical dengue, but incidence of dengue fever in the 2016 outbreak in Burkina Faso was also higher among teenagers and young adults $(4,6,32)$. In contrast, in the outbreak interval S3-S4, the FOI was similar across ages (13\%-34\%), possibly suggesting the emergence of a different serotype with little preexisting population immunity (20). Without much data on serotype-specific DENV incidence in Burkina Faso, previous studies and reports of ministry of health and World Health Organization investigations suggest that the 2016 outbreak was mainly caused by DENV-2 $(4,6,32)$.

Myalgia during S1-S2, arthralgia during S2-S3, and fatigue during S3-S4 were positively associated with seroconversion with statistical significance. Whereas cases of seroconversion may have been associated with either mild or subclinical illnesses, participants were asked whether they had experienced these symptoms in the interval since the previous survey. These symptoms are common in dengue illness; myalgia and arthralgia were listed in the 1997 World Health Organization dengue case classifications, and fatigue or lethargy in 2009 classifications (33-35). However, because these self-reported symptoms were recorded at intervals of 6-8 months, without prompt investigation to identify a cause, we cannot conclude that any associations with seroconversion are causal.

This study is subject to several limitations. First, the generalizability of this study is limited by participants all being recruited from the urban population in the capital city. The magnitude and patterns of DENV transmission may differ in other regions of Burkina Faso, including rural areas, with different age profiles, ecologic settings, or socioeconomic conditions. Also, although a large number of participants $(1,269)$ participated in all 4 serosurveys, a substantial number of original participants missed surveys. Those participants who missed $\geq 1$ surveys were more likely to be older than those who participated in all surveys. This shortfall could have led us to underestimate prevalence.

Second, our results were based on serologic testing using IgG ELISA. Further analyses using neutralization assays are planned, but no confirmatory testing was applied to verify the IgG results. For estimating dengue FOI, serologic cross-reaction with other flaviviruses affecting the observed dengue IgG rates is a commonly reported limitation $(36,37)$. Ultimately, this limitation could result in inaccurate seroprevalence and FOI estimates because antibodies against nondengue flaviviruses could have been detected $(15,38)$. In particular, Zika virus has been reported in Burkina Faso $(39,40)$, as have outbreaks of YF in 1998, 2003, and 2004 (41-43). Vaccine-induced YF antibodies could also result in interference with the specificity of IgG ELISA (38). In our data, at each of the serosurveys, $<5 \%$ of participants answered that they had received YF vaccination; although subject to recall bias, this rate is much lower than the coverage rate of $85 \%$ reported by the Expanded Program on Immunization in 2007 (42). Using self-reported YF vaccination history as a proxy for YF virus seropositivity, we found no difference in IgG positivity between participants reporting and not reporting vaccination, a finding supported by others $(38,44)$.

Despite the possible effect of cross-reactivity on dengue ELISAs, 2 studies reported a concordance level of $99 \%$ between the plaque reduction neutralization test (PRNT) and dengue IgG results $(37,45)$. Also, when IgG ELISA results were verified by using serologic data among 13,661 participants from 13 countries to estimate dengue FOI, IgG ELISA results were confirmed by $50 \%$ PRNT in $>97 \%$ of the IgGpositive samples (20). Samples from 277 healthy 
adults in a rural district in Malaysia were tested with the same ELISA test used in our study, and PRNT was performed on a subset of IgG-positive samples (46). Evidence of past infection was found in $75.5 \%$ (209/277) of participants and, of 96 samples randomly selected to undergo PRNT testing, all 96 were dengue-confirmed with $\geq 50 \%$ plaque reduction for DENV and showed that the detected antibodies were specific to dengue virus (46).

The sensitivity of this commercial DENV IgG indirect ELISA was reported as $99.2 \%$ and the specificity as $96.2 \%$ when compared with the hemagglutination inhibition (HI) method (47). A study compared performance of IgG ELISA to that of $\mathrm{HI}$ testing in a serosurvey of 327 children in Tahiti and reported sensitivity and specificity, as well as agreement between the 2 tests, to be $>83 \%$ for all DENV serotypes (24). Another study reported $90.9 \%$ sensitivity and 92.9\% specificity of the IgG indirect ELISA when compared with HI, also with a high correlation between the tests (48). Whereas those data are from outside of Africa, where a different and unknown composition of flaviviruses may be circulating, the results indicate a high degree of agreement between IgG ELISA and more confirmatory and better validated tests, such as HI and PRNT.

If cross-reactivity across other flaviviruses were to result in misclassifications, leading to a high rate of false positives, our findings would be overestimates of the true disease prevalence and FOI. However, given the high concordance between IgG and PRNT or $\mathrm{HI}$ assay results based on available data and because we analyzed data from paired serosurveys of participants who were IgG negative at enrollment, we believe that our results were minimally affected by this issue.

In conclusion, our estimates of both seroprevalence and FOI were comparable with those from dengue-endemic countries in the Americas. Repeated outbreaks indicate a considerable level of DENV transmission in Ouagadougou, but the extent of transmission and hyperendemicity needs to be further verified. Specifically, additional longitudinal evaluation with confirmatory tests and linked clinical evaluation of dengue fever in the general population in the region would be necessary to further validate our findings. Seroprevalence and FOI are important factors to be considered when making evidence-based decisions to implement interventions for prevention and control, including vaccine introduction. In the absence of other reliable data, our findings on dengue seroprevalence and FOI based on consecutive serosurveys provide practical evidence that could be used to support policy decisions.

This study was supported by funding from the Bill and Melinda Gates Foundation (grant no. OPP 1053432), as well as funding from the governments of Sweden, India, and South Korea. This research project was part of the "Community Research Studies and Interventions for Health Equity in Burkina Faso" program, funded by the Canadian Institutes of Health Research (ROH-115213). N.A. and T.E. were supported by award MR/R010161/1, which is jointly funded by the UK Medical Research Council (MRC) and the UK Department for International Development (DFID) under the MRC/DFID Concordat agreement and is also part of the European and Developing Countries Clinical Trials Partnership (EDCTP) -2 program, supported by the European Union.

\section{About the Author}

Dr. Lim is a research scientist in the Development and Delivery Unit of the International Vaccine Institute (IVI), Seoul, South Korea. She has been working as an epidemiologist in IVI for the Global Dengue and Aedes-Transmitted Diseases Consortium, previously the Dengue Vaccine Initiative, where she was in charge of field operations in Thailand, Colombia, Vietnam, Cambodia, Burkina Faso, Gabon, and Kenya.

\section{References}

1. World Health Organization. Global strategy for dengue prevention and control 2012-2020. Geneva: World Health Organization; 2012. Report no.: WHO/HTM/NTD/ VEM/2012.5 [cited 2018 Aug 18]. https://www.who.int/ denguecontrol/9789241504034

2. Amarasinghe A, Kuritsky J, Letson G, Margolis H. Dengue virus infection in Africa. Emerg Infect Dis. 2011;17:1349-54. https:/ / dx.doi.org/10.3201/eid1708.101515

3. Ministère de la Santé. Progress report on the investigation of suspected cases of dengue in the Central health region [in French]. Ouagadougou, Burkina Faso: Directorate of Disease Control; 2013.

4. World Health Organization. Dengue fever-Burkina Faso. Disease outbreak news: 2016 November 18 [cited 2018 Aug 18]. http://www.who.int/csr/don/18-november-2016dengue-burkina-faso/en/

5. World Health Organization. Dengue fever - Burkina Faso. Disease outbreak news: 2017 November 6 [cited 2018 Aug 18]. http://www.who.int/csr/don/6-november-2017dengue-burkina-faso/en/

6. Tarnagda Z, Cissé A, Bicaba B, Diagbouga S, Sagna T, Ilboudo A, et al. Dengue fever in Burkina Faso, 2016. Emerg Infect Dis 2018;24:170-2. https:/ / dx.doi.org/10.3201/ eid2401.170973

7. Ridde V, Agier I, Bonnet E, Carabali M, Dabiré K, Fournet F, et al. Presence of three dengue serotypes in Ouagadougou (Burkina Faso): research and public health implications. Infect Dis Poverty. 2016;5:23. https:/ /10.1186/s40249016-0120-2 
8. Collenberg E, Ouedraogo T, Ganame J, Fickenscher H, Kynast-Wolf G, Becher H, et al. Seroprevalence of six different viruses among pregnant women and blood donors in rural and urban Burkina Faso: a comparative analysis. J Med Virol. 2006;78:683-92. https://doi.org/10.1002/ jmv.20593

9. Lim J, Carabali M, Lee J-S, Lee K-S, Namkung S, Lim S-K, et al. Evaluating dengue burden in Africa in passive fever surveillance and seroprevalence studies: protocol of field studies of the Dengue Vaccine Initiative. BMJ Open. 2018;8:e017673. https://doi.org/10.1136/bmjopen-2017-017673

10. Messina J, Brady O, Scott T, Zou C, Pigott D, Duda K, et al Global spread of dengue virus types: mapping the 70 year history. Trends Microbiol. 2014;22:138-46. https://doi. org/10.1016/j.tim.2013.12.011

11. Brady OJ, Gething PW, Bhatt S, Messina JP, Brownstein JS, Hoen AG, et al. Refining the global spatial limits of dengue virus transmission by evidence-based consensus. PLoS Negl Trop Dis. 2012;6:e1760. https://doi.org/10.1371/journal. pntd. 0001760

12. Ministry of Health (Burkina Faso). Statistical yearbook 2016 [in French]. Ouagadougou, Burkina Faso: Ministry of Health, 2017 [cited 2019 Apr 1]. http:/ / ghdx.healthdata.org/record/ burkina-faso-health-statistical-yearbook-2016

13. World Population Review. Population of cities in Burkina Faso (2019) [cited 2019 October 3]. http:/ / worldpopulationreview.com/countries/burkina-faso-population/cities/

14. Rossier C, Soura A, Baya B, Compaoré G, Dabiré B, Dos Santos S, et al. Profile: the Ouagadougou Health and Demographic Surveillance System. Int J Epidemiol. 2012;41:658-66. https://doi.org/10.1093/ije/dys090

15. Tam CC, Tissera H, de Silva AM, De Silva AD, Margolis HS, Amarasinge A. Estimates of dengue force of infection in children in Colombo, Sri Lanka. PLoS Negl Trop Dis. 2013;7:e2259. https://doi.org/10.1371/journal.pntd.0002259

16. Egger JR, Ooi EE, Kelly DW, Woolhouse ME, Davies CR, Coleman PG. Reconstructing historical changes in the force of infection of dengue fever in Singapore: implications for surveillance and control. Bull World Health Organ. 2008;86:161-240. https://doi.org/10.2471/blt.07.040170

17. Bhavsar D. Modelling binary data. London: Chapman and Hall; 1991.

18. Bhavsar A, Tam C, Garg S, Jammy G, Taurel A, Chong S, et al. Estimated dengue force of infection and burden of primary infections among Indian children. BMC Public Health. 2019;19:1116. https://doi.org/10.1186/ s12889-019-7432-7

19. Prayitno A, Taurel A, Nealon J, Satari HI, Karyanti MR, Sekartini R, et al. . Dengue seroprevalence and force of primary infection in a representative population of urban dwelling Indonesian children. PLoS Negl Trop Dis. 2017; 11:e0005621. [Erratum in: PLoS Negl Trop Dis. 2018; 12:e0006467.] https:// doi.org/10.1371/journal.pntd.0005621

20. Nealon J, Bouckenooghe A, Cortes M, Coudeville L, Frago C, Macina D, et al. Dengue endemicity, force of infection, and variation in transmission intensity in 13 endemic countries. J Infect Dis. 2020:jiaa132. [Erratum in: J Infect Dis. 2020;222:341-2.] https:/ / doi.org/10.1093/infdis/ jiaa132.

21. Bhatt S, Gething PW, Brady OJ, Messina JP, Farlow AW, Moyes CL, et al. The global distribution and burden of dengue. Nature. 2013;496:504-7. https:/ / doi.org/10.1038/ nature12060

22. Jaenisch T, Junghanss T, Wills B, Brady OJ, Eckerle I, Farlow A, et al. Dengue expansion in Africa-not recognized or not happening? Emerg Infect Dis. 2014;20:e140487. http:/ / dx.doi.org/10.3201/eid2010.140487

23. Carabali M, Lim J, Velez D, Trujillo A, Egurrola J, Lee K, et al. Dengue virus serological prevalence and seroconversion rates in children and adults in Medellin, Colombia: implications for vaccine introduction. Int J Infect Dis. 2017;58:27-36. https:/ / doi.org/10.1016/j.ijid.2017.02.016

24. Chungue E, Marché G, Plichart R, Boutin J, Roux J. Comparison of immunoglobulin g enzyme-linked immunosorbent assay (IgG-ELISA) and haemagglutination inhibition (HI) test for the detection of dengue antibodies, prevalence of dengue IgG-ELISA antibodies in Tahiti. Trans R Soc Trop Med Hyg. 1989;83:708-11. https:/ / doi.org/ 10.1016/0035-9203(89)90404-5

25. Braga C, Luna CF, Martelli CM, de Souza WV, Cordeiro $\mathrm{MT}$, Alexander N, et al. Seroprevalence and risk factors for dengue infection in socio-economically distinct areas of Recife, Brazil. Acta Tropica. 2010;113:234-40. https://doi. org/10.1016/j.actatropica.2009.10.021

26. Teixeira MG, Barreto ML, Costa MCN, Ferreira LDA, Vasconcelos PFC, Cairncross S. Dynamics of dengue virus circulation: a silent epidemic in a complex urban area. Trop Med Int Health. 2002;7:757-62. https:/ / doi.org/10.1046/ j.1365-3156.2002.00930.x

27. Boni M, Mølbak K, Krogfelt K. Inferring the time of infection from serological data. In: Held L, Hens N, O'Neill P, Wallinga, J, editors. Handbook of infectious disease data analysis. Boca Raton (FL): CRC Press; 2019. p. 287-303.

28. Wongkoon $S$, Jaroensutasinee $M$, Jaroensutasinee $\mathrm{K}$. Distribution, seasonal variation and dengue transmission prediction in Sisaket, Thailand. Indian J Med Res. 2013; 138:347-53.

29. Tan K-K, Zulkifle T-I, Abd-Jamil J, Sulaiman S, Yaacob CN, Azizan NS, et al. Disruption of predicted dengue virus type 3 major outbreak cycle coincided with switching of the dominant circulating virus genotype. Infect Genet Evol. 2017:271-5. https://doi.org/10.1016/j.meegid.2017.07.008

30. Gubler DJ. Dengue and dengue hemorrhagic fever. Clin Microbiol Rev 1998;11:480-96.

31. Imai N, Dorigatti I, Cauchemez S, Ferguson NM. Estimating dengue transmission intensity from sero-prevalence surveys in multiple countries. 2015;9(4):e0003719. https:/ / doi.org/ 10.1371/journal.pntd.0003719

32. Lim JK, Seydou Y, Carabali M, Barro A, Dahourou DL, Lee KS, et al. Clinical and epidemiologic characteristics associated with dengue during and outside the 2016 outbreak identified in health facility-based surveillance in Ouagadougou, Burkina Faso. PLoS Negl Trop Dis. 2019; 13:e0007882. https://doi.org/10.1371/journal.pntd.0007882

33. World Health Organization. Dengue guidelines for diagnosis, treatment, prevention, and control: new edition. Geneva: The Organization; 2009.

34. World Health Organization. Dengue and severe dengue. Fact sheets 20192 February [cited 2019 May 12]. http:/ / www. who.int/news-room/fact-sheets/detail/dengue-and-severedengue

35. Hadinegoro SRS. The revised WHO dengue case classification: does the system need to be modified? Paediatr Int Child Health 2012;32 Suppl 1:33-8. https:/ / doi.org/ 10.1179/2046904712Z.00000000052

36. Oliveira RA, de Oliveira-Filho EF, Fernandes AI, Brito CA, Marques ET, Tenório MC, et al. Previous dengue or Zika virus exposure can drive to infection enhancement or neutralisation of other flaviviruses. Mem Inst Oswaldo Cruz. 2019;114:e190098. https:// doi.org/10.1590/ 0074-02760190098 
37. Garg S, Chakravarti A, Singh R, Masthi NRR, Goyal RC, Jammy GR, et al. Dengue serotype-specific seroprevalence among 5- to 10-year-old children in India: a communitybased cross-sectional study. Int J Infect Dis. 2017;54:25-30. https://doi.org/10.1016/j.ijid.2016.10.030

38. Santiago e Souza NC, Félix AC, de Paula AV, Levi JE, Pannuti CS, Romano CM. Evaluation of serological crossreactivity between yellow fever and other flaviviruses. Int J Infect Dis. 2019;81:4-5. https:/ / doi.org/10.1016/ j.ijid.2019.01.023

39. World Health Organization. Zika virus (ZIKV) classification table. Geneva: The Organization; 2018 [cited 2019 June 1]. https:/ / apps.who.int/iris/handle/ $10665 / 260419$

40. Majumder MS, Hess R, Ross R, Piontkivska H. Seasonality of birth defects in West Africa: could congenital Zika syndrome be to blame? F1000 Res. 2018;2018:159. https://doi.org/10.12688/f1000research.13858.2

41. Collenberg E, Ouedraogo T, Ganamé J, Fickenscher H, Kynast-Wolf G, Becher H, et al. Seroprevalence of six different viruses among pregnant women and blood donors in rural and urban Burkina Faso: a comparative analysis. J Med Virol. 2006;78:683-92. https:/ / doi.org/10.1002/ jmv.20593

42. World Health Organization. Yellow fever in Burkina Faso. 2008 [cited 2019 May 29]. https:/ / www.who.int/csr/ don/2008_11_03

43. Mutebi J-P, Barrett ADT. The epidemiology of yellow fever in Africa. Microbes Infect. 2002;4:1459-68. https:/ / doi.org/ 10.1016/s1286-4579(02)00028-x

44. da Silva-Nunes M, de Souza V, Pannuti C, Sperança M, Terzian A, Nogueira M, et al. Risk factors for dengue virus infection in rural Amazonia: population-based crosssectional surveys. Am J Trop Med Hyg 2008;79:485-94.

45. Sasmono R, Taurel A, Prayitno A, Sitompul H,

Yohan B, Hayati R, et al. Dengue virus serotype distribution based on serological evidence in pediatric urban population in Indonesia. PLoS Negl Trop Dis. 2018;12:e006616. https:// doi.org/10.1371/journal. pntd.0006616

46. Dhanoa A, Hassan S, Jahan N, Reidpath D, Fatt Q, Ahmad M, et al. Seroprevalence of dengue among healthy adults in a rural community in Southern Malaysia: a pilot study. Infect Dis Poverty. 2018;16:1. https:/ / doi.org/ 10.1186/s40249-017-0384-1

47. McBride WJ, Mullner H, LaBrooy JT, Wronski I. The 1993 dengue 2 epidemic in North Queensland: a serosurvey and comparison of hemagglutination inhibition with an ELISA. Am J Trop Med Hyg. 1998;59:457-61. https:/ / doi.org/ 10.4269/ajtmh.1998.59.457

48. Inoue S, Alonzo M, Kurosawa Y, Mapua C, Reyes J, Dimaano E, et al. Evaluation of a dengue IgG indirect enzyme-linked immunosorbent assay and a Japanese encephalitis IgG indirect enzyme-linked immunosorbent assay for diagnosis of secondary dengue virus infection. Vector Borne Zoonotic Dis. 2010;10:143-50.

Address for correspondence: Jacqueline K. Lim, Research Scientist, Global Dengue and Aedes-transmitted Diseases Consortium, International Vaccine Institute, SNU Research Park, Gwankak-ro 1, Gwanak-gu, Seoul 151-191, South Korea; email: kajlim@gmail.com 